\title{
Endurance Exercise Effects on Cardiac Hypertrophy in Mice
}

\author{
Gun-Soo Han, PhD ${ }^{1)}$ \\ 1) Department of Sports and Leisure Studies, College of Humanity, Daegu University: Jillyang, \\ Gyeongsan, Gyeongbuk 712-714, Republic of Korea
}

\begin{abstract}
Purpose] The purpose of this study was to investigate the effects of eight weeks of an endurance exercise training program on cardiac hypertrophy in mice. [Subjects] Male 129 SvJ/C57BL6 mice ( $\mathrm{n}=12$ ) were used. The exercised mice ran on a motor-driven treadmill five days per week for 40 minutes at a speed of $24 \mathrm{~m} / \mathrm{min}$ for eight weeks. All mice were weighed once a week to monitor excessive increases or decreases in weight. Peak weight was determined as the highest weekly recorded weight. Post-training weight was also taken on the day of final data collection. Following body weight measurement, the heart was excised from the body and weighed. The ratio of heart weight to body weight was calculated as an indicator of cardiac hypertrophy in the current study. Using an independent $t$ test, the ratio of heart weight to body weight was compared between the exercised mice and the sedentary mice. [Results] The results show that the untrained mice had a significantly greater heart weight to body weight ratio compared with the wild-type mice. There was also a significant difference in body weight between the exercised and sedentary groups. The ratio of heart weight to body weight was lower in the untrained mice, but no significance was observed. [Conclusion] Running on the motor- driven treadmill five days per week for 40 minutes at a speed of $24 \mathrm{~m} / \mathrm{min}$ for eight weeks did not increase in the ratio of heart weight to body weight in mice compared with the sedentary.

Key words: Cardiac hypertrophy, Endurance exercise, Treadmill
\end{abstract}

(This article was submitted May 15, 2013, and was accepted Jun. 24, 2013)

\section{INTRODUCTION}

Cardiac hypertrophy is a phenomenon with a genetic or environmental cause; however, it is not been yet completely understood. One of the most reasonable pieces of evidence is high blood pressure due to a narrowing heart valve. Another possible reason is physical stress such as endurance exercise training. A long-term exercise requires the heart to utilize and oxidize adequate amounts of fatty acids to function appropriately. Inducing cardiac hypertrophy is a physiological beneficial adaptation to exercise training. The oxidation of fatty acids in the mitochondria is the main process for providing adenosine triphosphate (ATP) for the heart, particularly during periods of fasting and prolonged exercise, even though the heart can utilize carbohydrates as energy substrates ${ }^{1}$. Long-chain fatty acids are the primary fuel source for the heart when the heart is in its normal resting condition. In other words, long-chain acids are the most important energy source, providing over $90 \%$ of cardiac energy ${ }^{2}$. For this rich source of potential energy to be used during exercise and fasting conditions, adipose tis-

Corresponding author. Gun-Soo Han (E-mail: gunsoo@ gmail.com)

C2013 The Society of Physical Therapy Science

This is an open-access article distributed under the terms of the Creative Commons Attribution Non-Commercial No Derivatives (by-ncnd) License $<$ http://creativecommons.org/licenses/by-nc-nd/3.0/>. sue triglycerides must first be hydrolyzed, and the resultant fatty acids must then be exported from adipose tissue and delivered to the tissue where they are oxidized ${ }^{3}$. Thus, endurance types of exercise induce a physiological change in the heart to adapt to the increased mechanical load ${ }^{4}$. Participating in exercise training induces cardiac hypertrophy ${ }^{5)}$, although it has not been identified. Therefore, the purpose of this study was to investigate whether an endurance exercise program for eight weeks would have an impact on the ratio of heart weight to body weight in mice compared with the sedentary mice.

\section{SUBJECTS AND METHODS}

All experiments were approved by the Institutional Animal Care and Use Committee of the University of Arkansas. Fifteen-week-old wild-type male 129 SvJ/C57BL6 mice $(\mathrm{n}=12)$ were used for this study. The mice were divided into two experimental groups (Exercise, $n=6$; Control, $n=6$ ). They were housed five animals per cage with a light-dark cycle of twelve hours and had access to laboratory rodent chow ad libitum. The exercised group ran on a motor-driven treadmill five days per week for 40 minutes at a speed of $24 \mathrm{~m} \cdot \mathrm{min}^{-1}$ for 8 weeks including a 5 -minute warm-up and cool down, respectively, at $10 \mathrm{~m} / \mathrm{min}$. The sedentary experimental group was left sedentary for the experiment. Before the training section, all animals were allowed a minimum 1-week familiarization of 15 minutes of exercise at 10-15 $\mathrm{m} / \mathrm{min}$ based on the animal status. During the final week of 
this study, the mice were randomly selected for the single bouts of exhaustive exercise. When a mouse was unable to keep up with the treadmill, the exercise session was terminated. At the end of the experimentation period, the mice were transported to the laboratory for data collection. Both groups of mice were anesthetized with sodium pentobarbital $\left(40 \mathrm{mg} / \mathrm{kg}^{-1}\right.$, ip). When anesthesia was confirmed, the hearts were rapidly removed from the animals and rinsed in ice-cold buffer containing $225 \mathrm{mM}$ mannitol, $75 \mathrm{mM}$ sucrose, $10 \mathrm{mM}$ MOPS, $10 \mathrm{mM}$ Tris HCL and $1 \mathrm{mM}$ EGTA at pH 7.2 (Buffer I). The connective tissue was then removed so that the tissue could be analyzed. The heart was minced on ice and placed back into a test tube to be weighed. All mice were weighed once a week to monitor for excessive increases or decreases in weight. Peak weight was determined as the highest weekly recorded weight. Post-training weight was also taken on the day of final data collection. An independent t-test was used to compare the exercise and control groups. All comparisons were made at the $\mathrm{p} \leq 0.05$ level of significance. Statistical analysis was conducted using StatView, Version 5.0.

\section{RESULTS}

Table 1 displays the results of the t-test for heart weight to body weight ratio. The untrained mice had a significantly greater heart weight to body weight ratio compared with the wild-type mice. There was also a significant difference in body weight between the two groups $(p<0.05)$. The ratio of heart weight to body weight was lower in the untrained mice, but no significance was observed.

\section{DISCUSSION}

Cardiac hypertrophy is a thickening of the heart muscle (myocardium) that results in a decrease in the size of the chamber of the heart, including the left and right ventricles. In other words, cardiac mass is increased in the response to applied physical stress. A common cause of cardiac hypertrophy is high blood pressure and heart valve stenosis. Even though there are many exercise benefits such as improving cardiac function and development of the skeletal system, exercise training induces changes in the cardiovascular system including oxygen delivery capacity and blood flow to the body ${ }^{6}$. The present study found that 8 weeks of exercise training could not induce significant cardiac hypertrophy in mice compared with sedentary mice. In other words, the results indicated that cardiac hypertrophy was less severe in the trained mice compared with the untrained mice. This finding indicates that exercise training for the mice did not provoke a change in cardiac function. This result is consistent with research conducted on the effects of treadmill running on cardiovascular phenotypes in transgenic mice ${ }^{7)}$. Endurance training has been proposed to have beneficial effects on oxidative phosphorylation through increased mitochondrial biogenesis ${ }^{8)}$. In this study, the trained mice ran more than the untrained mice during a single bout of treadmill running, although the difference in the ratio of heart weight to body weight was not significant. Advantages and disadvantages of different types of exercise training are
Table 1. Body weight, heart weight, ratio of heart weight to body weight, and run time after the endurance exercise training

\begin{tabular}{lcc}
\hline Variables & Trained $(\mathrm{n}=6)$ & Untrained $(\mathrm{n}=6)$ \\
\hline Body weight $(\mathrm{g})$ & $25.9 \pm 2.2$ & $29.3 \pm 1.6^{*}$ \\
Heart weight $(\mathrm{mg})$ & $140 \pm 8$ & $160 \pm 29^{*}$ \\
Ratio of heart weight $(\mathrm{mg})$ & $5.40 \pm 1$ & $5.46 \pm 1$ \\
to body weight $(\mathrm{g})$ & $40.1 \pm 9.4$ & $33.5 \pm 7.3^{*}$ \\
Run time (min) &
\end{tabular}

Values are means \pm SE. ${ }^{*}$ Significantly different $(p<0.05)$ from the trained mice

controversial. Based on the results of this study, the intensity and frequency of the exercise program were not enough to increase the ratio of heart weight to body weight. One limitation of this study was that due to physical and psychological stress from forced treadmill running, it may have been difficult to motivate the mice to keep running for the scheduled time. However, this stress may induce a change in the endocrine system in animals. This may potentially obscure the response to exercise training. An interesting finding in this study is that the sedentary mice showed a greater heart weight due to high body weight compared with the trained mice. This physiological advantage could not alter the untrained mice, decreasing run time. According to a study conducted by Maron and Pelliccia9), an increase in left ventricular mass is commonly observed endurance exercise training. The present study used endurance exercise training as an independent variable, but it is necessary for future researchers to use other factors influencing endurance exercise training-induced cardiac hypertrophy including age, sex, and exercise type and intensity. Also, there are many different ways to measure cardiac hypertrophy. We used the ratio of heart weight to body weight, which is the most common index of cardiac hypertrophy. This method has a few assumptions. Therefore, further study should consider ultrasound echocardiography and cardiomyocytes for better results ${ }^{10)}$.

\section{ACKNOWLEDGEMENT}

This research was supported by the Daegu University Research Grant, 2012.

\section{REFERENCES}

1) Mason KE, Stofan DA, Szweda LI: Inhibition of very long chain acyl-CoA dehydrogenase during cardiac ischemia. Arch Biochem Biophys, 2005, 437: 138-143. [Medline] [CrossRef]

2) Maín-García J, Goldenthal MJ: Mitochondrial centrality in heart failure. Heart Fail Rev, 2008, 13: 137-150. [Medline] [CrossRef]

3) Horowitz JF: Fatty acid mobilization from adipose tissue during exercise. Trends Endocrinol Metab, 2003, 14: 386-392. [Medline] [CrossRef]

4) Amaral SL, Silveira NP, Zorn TM, et al.: Exercise training causes skeletal muscle venular growth and alters hemodynamic responses in spontaneously hypertensive rats. J Hypertens, 2001, 19: 931-940. [Medline] [CrossRef]

5) Hambrecht R, Adams V, Erbs S, et al.: Regular physical activity improves endothelial function in patients with coronary artery disease by increasing phosphorylation of endothelial nitric oxide synthase. Circulation, 2003, 
107: 3152-3158. [Medline] [CrossRef]

6) Wei M, Gibbons LW, Mitchell TL, et al.: The association between cardiorespiratory fitness and impaired fasting glucose and type 2 diabetes mellitus in men. Ann Intern Med, 1999, 130: 89-96. [Medline] [CrossRef]

7) Fewell JG, Osinska H, Klevitsky R, et al.: A treadmill exercise regimen for identifying cardiovascular phenotypes in transgenic mice. Am J Physiol, 1997, 273: H1595-H1605. [Medline]
8) Wen T, Diaz F, Hernandez D, et al.: Endurance exercise is protective for mice with mitochondrial myopathy. J Appl Physiol, 1997, 106: 1712-1719.

9) Maron BJ, Pelliccia A: The heart of trained athletes: cardiac remodeling and the risk of sports, including sudden death. Circulation, 2006, 114: 1633-1644. [Medline] [CrossRef]

10) Wang $\mathrm{Y}$, Wisloff U, Kemi OJ: Animal models in the study of exerciseinduced cardiac hypertrophy. Physiol Res, 2010, 59: 633-644. [Medline] 